\title{
Interplay among spin, orbital effects and localization in a GaAs two-dimensional electron gas in a strong in-plane magnetic field
}

\author{
B. A. Piot $^{1}$, D. K. Maude ${ }^{1}$, U. Gennser ${ }^{2}$, A. Cavanna ${ }^{2}$, and D. Mailly ${ }^{2}$ \\ 1 Laboratoire National des Champs Magnétiques Intenses, \\ Grenoble High Magnetic Field Laboratory, Centre National de la Recherche Scientifique, \\ 25 Avenue des Martyrs, F-38042 Grenoble, France and \\ 2 Laboratoire de Photonique et de Nanostructures, \\ Centre National de la Recherche Scientifique, Route de Nozay, 91460 Marcoussis, France
}

(Dated: December 5, 2018)

\begin{abstract}
The magnetoresistance of a low carrier density, disordered GaAs based two-dimensional (2D) electron gas has been measured in parallel magnetic fields up to $32 \mathrm{~T}$. The feature in the resistance associated with the complete spin polarization of the carriers shifts down by more than $20 \mathrm{~T}$ as the electron density is reduced, consistent with recent theories taking into account the enhancement of the electron-electron interactions at low densities. Nevertheless, the magnetic field for complete polarization, $B_{p}$, remains 2-3 times smaller than predicted for a disorder free system. We show, in particular by studying the temperature dependance of $B_{p}$ to probe the effective size of the Fermi sea, that localization plays an important role in determining the spin polarization of a $2 \mathrm{D}$ electron gas.

PACS numbers: 73.43.Qt, 73.40.Kp
\end{abstract}

\section{INTRODUCTION}

The subtle physics of $2 \mathrm{D}$ systems subjected to a parallel magnetic field has stimulated increasing interest over the last decade. Historically, the selective coupling of the in-plane magnetic field to the electronic spin degree of freedom has been used to probe the influence of many body effects on the spin polarization and the ground state of the 2D electron gas (2-DEG). In practice, the application of a parallel magnetic field progressively lifts the spin degeneracy of the Fermi liquid until it becomes fully spin polarized. In transport experiments for example, the associated reduction in the screening of charged impurities leads to an increase of the longitudinal resistance followed by a saturation or a 'kink' signaling the complete spin polarization of the $2 \mathrm{D}$ system $1,2,3$ The magnetic field $B_{p}$ required to achieve complete polarization is a subtle quantity depending on the magnetic field dependent susceptibility, which is itself renormalized by many body effects (see e.g. Ref. [4]). An examination of the dependence of $B_{p}$ on different physical parameters such as carrier density, disorder, and temperature, has mostly been carried out in $\mathrm{Si}$ and p-GaAS based 2D systems, leaving the otherwise widely investigated n-GaAs 2DEG relatively unexplored concerning these issues.

However, it was recently emphasized that in non-ideal 2D systems which have a significant width, which is particularly relevant for n-GaAs, the in-plane magnetic field also couples to the orbital motion, and can considerably modify the physics involved, changing the effective disorder of the system,,$\frac{5}{\underline{6}}$ even stabilizing new phases $\frac{6}{6}$ It is therefore important to characterize the various physical effects generated by the presence of a parallel magnetic field, in order to gain a better understanding of the influence of electron-electron interactions on the ground state properties of the 2-DEG.
In this work, we report on experiments where a large in-plane magnetic field is applied to a $2 \mathrm{D}$ electron gas in GaAs, which reveal the interplay between disorder, spin and, orbital physics. Transport measurements under parallel magnetic fields up to $32 \mathrm{~T}$ show that, at sufficiently low electron density, the signatures of orbital, spin effects and localization coexist. Such a coexistence of distinct phenomena in a 2D system has to our knowledge not been previously reported. The magnetoresistance kink, associated with the complete spin polarization of the 2-DEG, is observed for magnetic fields up to $B_{p} \sim 30 \mathrm{~T}$. The observed reduction of $B_{p}$ of more than $20 \mathrm{~T}$ with decreasing electron density (and mobility) is in good agreement with the predicted enhancement of the spin susceptibility at low density due to electron-electron interactions. However, the absolute value of $B_{p}$ remains 2-3 times smaller than the calculated value at $T=0 K$ for a disorder-free system, and extrapolate to $B_{p}=0$ at a large, non zero electron density. This behavior is attributed to the localization of electrons in our disordered 2-DEG. $B_{p}$ shows a strong increase with temperature, consistent with a reduction of the spin susceptibility. A simple model yields an estimate of the density of delocalized states which is significantly smaller than the total electron density, confirming the role played by disorder in the observed phenomenon. In the frozen spin regime, the resistivity $\rho$ dramatically increases with a $\ln (\rho) \propto B^{2}$ dependence, in agreement with theory taking into account the orbital coupling to the parallel field.

\section{EXPERIMENTAL DETAILS}

The sample studied here is a volume-doped $\mathrm{Al}_{x} \mathrm{Ga}_{1-x} \mathrm{As} / \mathrm{GaAs}$ heterojunction (HJ), patterned into a Hall bar. The electron density $n_{s}$ was varied by 
the application of a gate voltage enabling us to tune the density between $4.2 \times 10^{10} \mathrm{~cm}^{-2}$ and $1.74 \times 10^{11} \mathrm{~cm}^{-2}$ so that the corresponding interaction parameter $r_{s}$ spans the range $3>r_{s}>1.5$. At zero gate voltage the mobility is $38 \mathrm{~m}^{2} / V s$, and decreases down to $\sim 2 \mathrm{~m}^{2} / V s$ in the low density regime (see Table \). The transport measurements were performed with a standard low frequency lock-in technique for temperatures between $1.17 \mathrm{~K}$ and $4.2 \mathrm{~K}$, under magnetic fields up to $32 \mathrm{~T}$ produced by a resistive magnet. The $2 \mathrm{D}$ electron plane was aligned parallel to the magnetic field direction by using an in-situ rotation stage to minimize the Hall voltage, with an accuracy estimated to be better than $1^{\circ}$. In the geometry used, the current along the Hall bar was always perpendicular to the applied magnetic field. The physical sample parameters are summarized for selected densities in Table I. Here the carrier densities are determined from the gate voltage versus carrier density dependence determined (for the same cooldown) in perpendicular magnetic field from the Hall voltage and/or the Shubnikov de Haas effect.

\section{RESULTS AND DISCUSSION}

\section{A. Spin-determined magnetoresistance}

In Fig 1 we plot the longitudinal resistivity $\rho$ as a function of the parallel magnetic field for different electron densities in the low density region. A number of different magnetic field regimes are observed over the range studied [0-32T]. The resistivity first increases as $\rho \propto B^{2}$ (as observed in Refs. [1,2]) until a kink emerges at a densitydependent magnetic field. This kink has been shown to be associated with the complete spin polarization of the system $\underline{3}$ eventually achieved at sufficiently high parallel magnetic field. The rich physics lying behind this quantity will be discussed throughout this paper. In 2DEG's in Si-MOSFET's, in which a similar feature was first reported,$\frac{1,2}{,}$ a saturation of the resistance is usually observed once full spin polarization occurs. Here, the resistance does not saturate after the kink but smoothly increases before rising exponentially, a behavior we attribute to the non-negligible thickness of the 2-DEG in GaAs making orbital coupling to the parallel magnetic field possible ${ }^{\frac{7}{3}}$ We stress this behaviour, which will be discussed in section $\amalg$ IIB, is qualitatively and quantitatively different from the initial $\rho \propto B^{2}$ increase.

TABLE I: Parameters of the gated GaAs HJ .Electron density $\left(n_{s}\right)$, zero field resistivity $\left(\rho_{0}\right)$, mobility $(\mu)$ and magnetic field for full polarization $\left(B_{p}\right)$ all measured at $T=1.17 \mathrm{~K}$.

\begin{tabular}{c|ccc}
$n_{s}\left(\mathrm{~cm}^{-2}\right)$ & $\rho_{0}(k \Omega / \square)$ & $\mu\left(m^{2} / V s\right)$ & $B_{p}(\mathrm{~T})$ \\
\hline $5.69 \times 10^{10}$ & 2.43 & 4.53 & 26.0 \\
$5.14 \times 10^{10}$ & 3.86 & 3.15 & 18.3 \\
$4.76 \times 10^{10}$ & 5.70 & 2.30 & 11.8 \\
$4.31 \times 10^{10}$ & 8.01 & 1.81 & 6.2
\end{tabular}

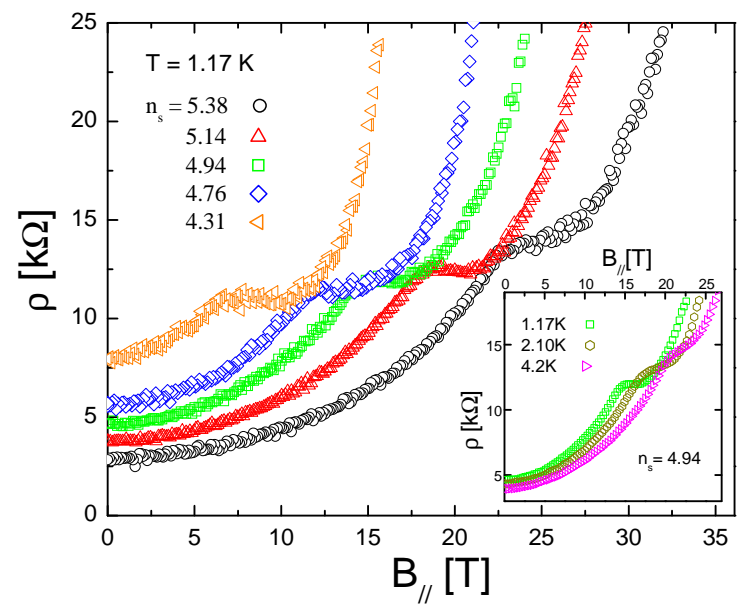

FIG. 1: (Color online) Longitudinale resistivity $\rho$ as a function of the parallel magnetic field for different electron densities $n_{s}$ in $10^{10} \mathrm{~cm}^{-2} . T=1.17 \mathrm{~K}$. Inset: temperature dependance of $\rho\left(B_{\|}\right)$at $n_{s}=4.94 \times 10^{10} \mathrm{~cm}^{-2}$.

From the magnetoresistance traces, we can extract the field $B_{p}$ at which a kink occurs in the resistivity. In Fig. 2(a), we plot the values of this field at $T=1.17 \mathrm{~K}$ and $T=4.2 \mathrm{~K}$ as a function of the electron density, together with data from Refs. [3,8] obtained at lower density in high mobility n-GaAs $2 \mathrm{D}$ systems. In a single electron picture, for an ideal 2-DEG, the condition for the full polarization of the Fermi sea is simply $g^{*} \mu_{B} B \geq 2 E_{F}$, where $g^{*}$ is the electronic effective bare g-factor, $\mu_{B}$ the Bohr magneton and $E_{F}$ the Fermi energy. Due to the strong enhancement of the g-factor by electron-electron exchange interactions at low density, the magnetic field for full spin polarization is actually much smaller. The calculation of this quantity is a challenging task since it requires modelling electron-electron interactions in the strongly interacting regime. Furthermore, the polarization dependance of the exchange interaction leads to a spin susceptibility which evolves non linearly with the magnetic field, thus affecting the polarization process $\underline{4}$ We plot in Fig.2(a) the critical magnetic field for full polarization at $T=0 K$ in the single electron picture (solid black line), together with recent theoretical calculations including many-body effects using different methods, respectively Random Phase Approximation (RPA) 4 and Quantum Monte Carlo Calculation (QMC) $\underline{\underline{9}}$

Our data at $1.17 \mathrm{~K}$ shows a dramatic reduction of $B_{p}$ as the density is lowered, the latter decreasing by $21 \mathrm{~T}$ in the range $[4.25-5.69] \times 10^{10} \mathrm{~cm}^{-2}$. This reduction with decreasing density is in good agreement with the $T=0 K$ predictions of Ref. [4] , as well as the predictions of Ref. [9], where $B_{p}$ also drops by about $20 \mathrm{~T}$ over the same density range. However, there is a global offset compared to theory, with the measured $B_{p}$ being about 2 or 3 times smaller than predicted. Furthermore, the low temperature $B_{p}$ versus $\left(n_{s}\right)$ trend extrapolates to zero at a density of about $\sim 3.5 \times 10^{10} \mathrm{~cm}^{-2}$, apparently suggest- 

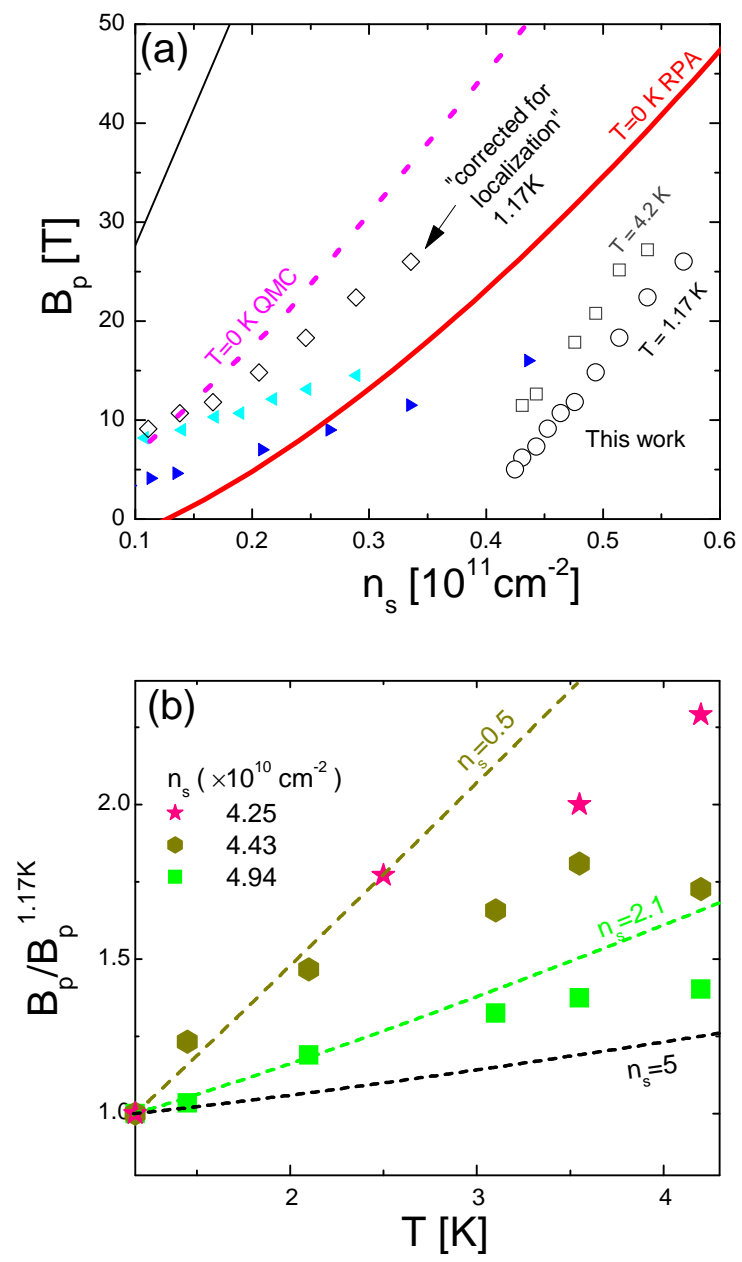

FIG. 2: (Color online) (a) Complete polarization field $B_{p}$ as a function of the electron density. Data at $T=1.17 \mathrm{~K}$ and $T=4.2 K$ from this work (open circles and open squares, respectively), and at $T=25 \mathrm{mK}$ from Refs. 3 8] (solid left and right triangles, respectively.) Data at $T=1.17 \mathrm{~K}$ plotted as a function of the "corrected" electron density (diamonds) (see text). Theoretical $T=0 K$ calculations of the magnetic field for full spin polarization in a single particle picture (solid line) and including many-body effects: $\mathrm{RPA}^{4}$ (thick solid line), and $\mathrm{QMC}^{9}$ (dotted line) (see text). (b) Temperature dependence of $B_{p}$ normalized with respect to the value at $1.17 \mathrm{~K}$, measured for different densities given in $10^{10} \mathrm{~cm}^{-2}$ (symbols). Calculation in a single particle picture for $n_{s}=0.5,2.1$ and $5 \times 10^{10} \mathrm{~cm}^{-2}$ (dotted line) (see text).

ing the appearance of a ferromagnetic state, whereas no ferromagnetic state has ever been observed in GaAs at these densities in much cleaner samples, even at mK temperature (see e.g. Ref. [10]). From a theoretical point of view, this transition is also expected for much lower densities (higher $r_{s}$ parameter) (see e.g. Refs. [11,12]). We believe that claims of a finite density ferromagnetic instability based only on the extrapolation of the $B_{p}\left(n_{s}\right)$ curves to zero magnetic field are misleading.

We argue that the physics that can account for these observations lies in the influence of disorder which has so far been neglected. The influence of disorder on the spin polarization has already been discussed for $\mathrm{Si}$ MOSFET's $\stackrel{13,14}{=}$ In Ref. [14], the field at which the magnetoresistance saturates was found to be sample dependent, increasing with the sample mobility. It was subsequently argued that the magneto resistance saturation actually reflects complete spin polarization of the nonlocalized carriers only,$\frac{15}{\mathfrak{}}$ so that the value of $B_{p}$ is very sensitive to the number of localised states in the system. At zero perpendicular magnetic field, a "2-component model", where a certain fraction of the electron are considered "bound" to impurities (i.e. localized), was introduced in Ref. [16] in the context of the 2D Metal-toInsulator transition (MIT), giving good agreement with experimental results. In our disordered GaAs 2-DEG, in which a significant fraction of electrons are localized, the Fermi sea which the magnetic field "has to polarize" is then effectively smaller, resulting in an experimental $B_{p}$ smaller than the one predicted by theories neglecting localization.

Additionally, as the density is reduced from $5.69 \times$ $10^{10} \mathrm{~cm}^{-2}$ to $4.25 \times 10^{10} \mathrm{~cm}^{-2}$ the electron mobility in our sample drops by a factor of nearly 3 (see Table 【). The associated decrease of the conductivity $\sigma_{0}=1 / \rho_{0}$ at $\mathrm{T}=1.17 \mathrm{~K}$ as a function of the electron density can be well described using the percolation model for the apparent MIT (see e.g. $\stackrel{17}{\underline{7}}$ ), where $\sigma_{0} \propto\left(n_{s}-n_{c}\right)^{p}$, with $p=1.31$ the conductivity exponent and $n_{c}$ the critical density for the MIT. Using $n_{c}$ as the only adjustable parameter, one

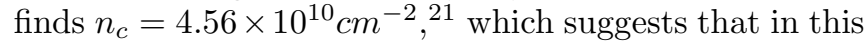
density region a significant proportion of the electronic states become localized, consistent with $B_{p}$ falling to zero in this region.

We believe these disorder effects are not observed in the data of Refs. [3,8] because of the much higher mobility (at least an order of magnitude) of the sample investigated therein. In the latter work, the experimental data and the theoretical predictions merge as density is lowered (see Tutuc et al. data in Fig[2(b)). It has been suggested that this behavior can be understood by taking into account the effective mass increase due to the orbital coupling to the parallel field in a 2-DEG with finite thickness. The increased effective mass leads to a smaller Fermi energy and thus a reduction of $B_{p}$. In such a picture the deviation from the theory for an ideal 2DEG is more pronounced at high electron density where the parallel magnetic fields involved are larger. Such a behavior is not observed in our data, in which the discrepancies between theory and experiment only weakly depend on density and are actually slightly more pronounced at lower densities. This confirms that in our case the $B_{p}$ reduction is a disorder-induced phenomenon rather than a finite thickness effect, the latter being most likely overwhelmed by the dramatic effect of electron localization (weaker in Refs. [3, 8$]$ ).

The effect of temperature on the magnetoresistance is shown for a density of $4.94 \times 10^{10} \mathrm{~cm}^{-2}$ in the inset of Fig,1, where a strong increase of $B_{p}$ is observed between 
$1.17 \mathrm{~K}$ and $4.2 \mathrm{~K}$. The evolution of $B_{p}$ with respect to its value at $T=1.17 \mathrm{~K}$, is plotted as a function of temperature in Fig 2(b). As can be seen, in this temperature region, $B_{p}$ follows a sub-linear variation with $\mathrm{T}$, the initial fast increase progressively slowing down at higher temperature. This trend differs from the temperature dependence of $B_{p}$ in the very low temperature regime recently observed for a $2 \mathrm{D}$ hole gas $\underline{18}$ The initial enhancement of $B_{p}$ becomes more and more pronounced as the density is decreased in the range $[4.25-5.14] \times 10^{10} \mathrm{~cm}^{-2}$ (only 3 densities are shown in Fig 2(b) for clarity). The primary influence of temperature at a given magnetic field is to reduce the electron spin polarization. In a very simple single electron picture, the spin polarization $S$ of the 2D Fermi sea in a parallel magnetic field is given by

$$
\begin{aligned}
& S\left(n_{s}, \Delta, E_{F}, T\right)= \\
& \frac{m^{*}}{2 \pi \hbar^{2} n_{s}} \int_{0}^{\infty} F D\left(E, E_{F}, T\right) \Phi(E) d E \\
& \quad-\frac{m^{*}}{2 \pi \hbar^{2} n_{s}} \int_{\Delta}^{\infty} F D\left(E, E_{F}, T\right) \Phi(E-\Delta) d E,
\end{aligned}
$$

where $F D$ is the Fermi distribution function, $\Phi$ is a Heaviside step function, $\Delta$ the spin gap separating the spin up and down subbands, $m^{*}$ the electron effective mass, and $E_{F}$ the magnetic field and temperaturedependent Fermi energy. One expects the magnetic field $B_{p}$, corresponding to $S=1$, to increase with temperature to compensate for the polarization reduction induced by the smearing of the Fermi distribution at the Fermi level.

Calculations of $B_{p}(T)$ normalized with respect to its value at $1.17 \mathrm{~K}$ obtained using this formalism are shown by the dotted lines in Fig.2(b), for different electron densities. At higher electron density, the Fermi sea is more robust with respect to temperature so that the $B_{p}$ enhancement is less important, in qualitative agreement with our experimental results $\frac{19}{9}$ Intriguingly, the quantitative temperature dependence corresponds more to the expected dependence for low density systems $\left(n_{s} \sim 0.5-2.1 \times 10^{10} \mathrm{~cm}^{-2}\right)$. We take this as evidence that only a fraction of the total electron density contributes to the effective "delocalized" Fermi sea responsible for the thermal effects, reinforcing our "disorder-based" interpretation of the reduced $B_{p}$ values. Comparing the experimental and theoretical low temperature dependence, we can estimate the number of delocalized states in our system. For instance, for $n_{s}=4.94 \times 10^{10} \mathrm{~cm}^{-2}$ (solid squares in Fig 2(b)), the electron density that best reproduces the low temperature trend using our model is $=2.1 \times 10^{10} \mathrm{~cm}^{-2}$. This suggests that only $40 \%$ of the electrons are delocalized at this density. For a total density of $n_{s}=4.25 \times 10^{10} \mathrm{~cm}^{-2}$ (hexagons in Fig 2(b)) our model yields a density of only $=0.5 \times 10^{10} \mathrm{~cm}^{-2}$, meaning $90 \%$ of the states have become localized, consistent with the proximity of the MIT in this density region $\underline{20}$ We can use the estimated density of delocalized electrons as a function of $n_{s}$ and re-plot the experimental $B_{p}$ at

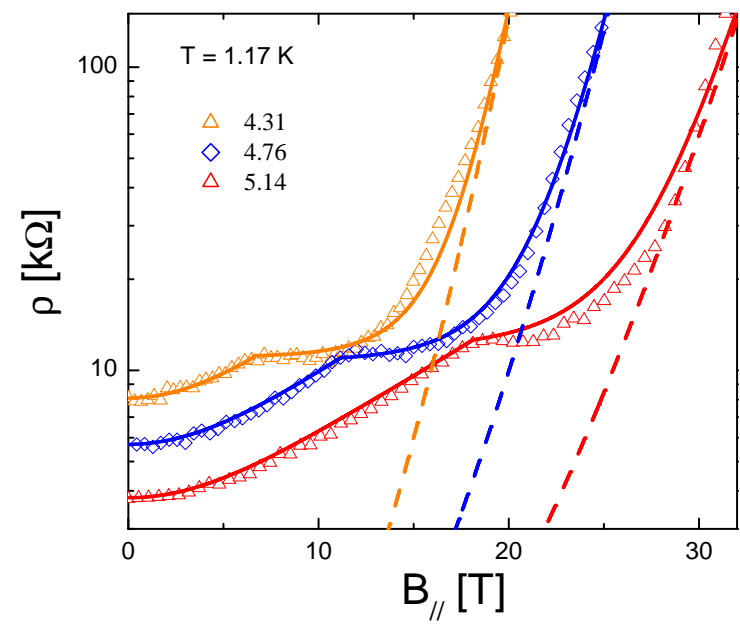

FIG. 3: (Color online) Resistivity $\rho$ in the high parallel field regime at $T=1.17 \mathrm{~K}$, for different densities $n_{s}$ in $\times 10^{10} \mathrm{~cm}^{-2}$ (symbols). $\ln (\rho) \propto B^{2}$ (dashed lines) and qualitative trend combining spin and orbital effects (solid lines) (see text).

$1.17 \mathrm{~K}$ as a function of these values (diamonds in Fig 2 a), which gives a better quantitative agreement with theory. We stress that, unlike the original data set (open circles), this corrected data set does not extrapolate to $B_{p}=0$ for a non zero carrier density (not shown). There is therefore no evidence of a ferromagnetic transition once localization is taken into account.

\section{B. High magnetic field behavior}

We now turn to the description of the "frozen-spin" regime where the system is fully polarized. As can be seen in Fig 1, for $B>B_{p}$, the resistance increases slowly before rising abruptly as the magnetic field is further increased. We stress that in this case, as the spin degree of freedom is frozen, the physical origin of this phenomenon cannot be spin related. In Fig 3 we focus on the high field behavior of the resistance by plotting the resistivity $\rho$ on a $\log$ scale. In the high field limit, the resistivity asymptotically follows an exponential power law, $\ln (\rho) \propto \alpha(B)^{2}$, where $\alpha$ increases monotonically as the density is reduced (dashed lines in Fig $\underline{3}$ ). The observed power law is qualitatively consistent with an increased intersubband scattering due to the orbital coupling to the parallel field, for which an identical magnetic field depen-

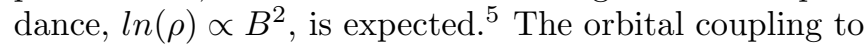
the in-plane magnetic field can be thought of as a parallel field induced effective 2D to 3D crossover in our low density quasi-2D system. The fact that the coefficient $\alpha$ increases at low density is consistent with this effect being stronger as the system becomes more dilute $\underline{\underline{\underline{5}}}$ One can combine these orbital effects with the initial $\rho \propto B^{2}$ "spin trend" to try to reproduce the general behavior of the resistance. For this we use the following expression for $\rho, \rho=\rho_{0}+\beta B^{2}+\gamma e^{\alpha B^{2}}$, where the second and third 
term corresponds to spin and orbital contributions to the resistance respectively. The "spin term" is set to $\beta B_{p}{ }^{2}$ for $B>B_{p}$ to account for the quench of spin effects, and $\beta=0.024-0.065$ increases with decreasing density, consistent with spin polarization growing faster at lower electron density. The "orbital term" is determined from the high field limit, with $\gamma=0.1$ (independent of density) and $\alpha=0.007-0.018$ for our density range. The obtained behaviour is plotted as solid lines in Fig 3 and reproduces the experimental data very well leading further support to our simple model.

\section{CONCLUSION}

In conclusion, we have shown that the physics of GaAs $2 \mathrm{D}$ electron systems under a strong parallel magnetic field is at low electron density determined by an interplay between spin, localization and orbital effects. The magnetic field at which complete spin polarization occurs is dramatically reduced both by electron localization and electron-electron interaction enhanced at low carrier density. The large value of the extrapolated carrier density for full spin polarization at zero magnetic field simply reflects the large number of localized electrons present in disordered systems and should under no circumstances be interpreted as evidence for a ferromagnetic instability.

\section{Acknowledgments}

The work at GHMFL was partially supported by the European 6th Framework Program under contract number RITA-CT-3003-505474
1 T. Okamoto, K. Hosoya, S. Kawaji, and A. Yagi, Phys. Rev. Lett. 82, 3875 (1999).

2 S. A. Vitkalov, H. Zheng, K. M. Mertes, M. P. Sarachik, and T. M. Klapwijk, Phys. Rev. Lett. 85, 2164 (2000).

3 E. Tutuc, S. Melinte, and M. Shayegan, Phys. Rev. Lett. 88, 036805 (2002).

${ }^{4}$ Y. Zhang and S. D. Sarma, Phys. Rev. Lett. 96, 196602 (2006).

5 S. Das Sarma and E. H. Hwang, Phys. Rev. Lett. 84, 5596 (2000).

${ }^{6}$ B. A. Piot, Z. Jiang, C. R. Dean, L. W. Engel, G. Gervais, L. N. Pfeiffer, and K. W. West, Nat Phys 4, 936 (2008).

7 S. Das Sarma and E. H. Hwang, Phys. Rev. B 72, 035311 (2005).

8 E. Tutuc, S. Melinte, E. P. De Poortere, M. Shayegan, and R. Winkler, Phys. Rev. B. 67, 241309(R) (2003).

9 A. L. Subaşi and B. Tanatar, Phys. Rev. B 78, 155304 (2008).

10 J. Zhu, H. L. Stormer, L. N. Pfeiffer, K. W. Baldwin, and K. W. West, Phys. Rev. Lett. 90, 056805 (2003).

11 C. Attaccalite, S. Moroni, P. Gori-Giorgi, and G. B. Bachelet, Phys. Rev. Lett. 88, 256601 (2002).

12 Y. Zhang and S. Das Sarma, Phys. Rev. B 72, 115317 (2005).

13 S. A. Vitkalov, M. P. Sarachik, and T. M. Klapwijk, Phys. Rev. B 65, 201106 (2002).
14 V. M. Pudalov, G. Brunthaler, A. Prinz, and G. Bauer, Phys. Rev. Lett. 88, 076401 (2002).

15 V. T. Dolgopolov and A. Gold, Phys. Rev. Lett. 89, 129701 (2002).

16 S. Das Sarma and E. H. Hwang, Phys. Rev. Lett. 83, 164 (1999)

17 S. He and X. C. Xie, Phys. Rev. Lett. 80, 3324 (1998).

18 X. P. A. Gao, G. S. Boebinger, J. A. P. Mills, A. P. Ramirez, L. N. Pfeiffer, and K. W. West, Phys. Rev. B 73, 241315 (2006).

19 We note that a sub-linear $B_{p}(T)$ trend is however not expected in our simple model, suggesting some more complexe effects are taking place at higher temperature.

20 The ability of our single particle model to determine the actual fraction of localized states is limited by the presence of many body effects. Simple considerations based on selfconsistent exchange enhancement of the spin gap show that neglecting these effects leads to an overestimation of the fraction of localized states.

21 It is important to stress here that a precise location of the MIT cannot be inferred from our $\mathrm{T}=1.17 \mathrm{~K}$ data. The critical density $n_{c}$ we quote here is only an approximation of the zero temperature value not taking into account the temperature dependence of the conductivity exponent, $\mathrm{p}$. 